\title{
ON JACKSON TYPE INEQUALITY IN ORLICZ CLASSES
}

\author{
Konstantin RUNOVSKI
}

\begin{abstract}
It is shown that Jackson type inequality fails in the Orlicz classes $\varphi(L)$ if $\varphi(x)$ differs essentially from a power function of any order.
\end{abstract}

\section{Introduction}

As is known a best approximation to a given $2 \pi$-periodic function in $L_{p}, 0<p \leq+\infty$, by trigonometric polynomials of order at most $n$ can be estimated by its $L_{p^{-}}$modulus of continuity with argument $(n+1)^{-1}$. This result is called the direct theorem of Approximation Theory or Jackson type inequality in honour of D. Jackson who proved it for continuous metric $(p=+\infty)$. Afterwards it was extended to the case of an arbitrary Banach space of $2 \pi$-periodic functions, where translation is a continuous isometry (see, for instance, [1]). The quasinormed case $0<p<1$ can be found in [2]. At present there is an enormous number of works dedicated to various generalizations of Jackson type inequality that deal with several variables, higher order moduli of smoothness and so forth. However, for a long time nothing was known about similar results in the setting of Orlicz classes $\varphi(L)$, where $\varphi(x)$ differs essentially from a power function of any order. As far as we know it was expected Jackson type inequality would be valid for comparatively wide set of functions $\varphi(x)$. To our surprise, it is not. In fact, the main result of this paper is that if $\varphi(x)$ decreases to 0 for $x \rightarrow+0$ or increases to $+\infty$ for $x \rightarrow+\infty$ slower than a power function of an arbitrary order, Jackson type inequality fails. Moreover, for such $\varphi(x)$ the modulus of continuity turns out in general to be unfit to estimate the rate of approaching 0 of a best trigonometric

2000 Mathematics Subject Classification: 42A10, 42A15.

Servicio de Publicaciones. Universidad Complutense. Madrid, 2001 
approximation in $\varphi(L)$ in the sense that the inequality remains false even after replacing $(n+1)^{-1}$ by an arbitrary sequence of positive numbers $\left\{\sigma_{n}\right\}_{n=1}^{+\infty}$ that goes to 0 for $n \rightarrow+\infty$.

We prove our result not only for the trigonometric system, but also for more general class of systems we have called non-localized. It will be shown that this class contains all systems of $2 \pi$-periodic analytic functions. The property of "non-locality" makes clear the difference between the trigonometric system and the system of piece-wise constant functions, for which Jackson type inequality holds in $\varphi(L)$, where $\varphi(x)$ satisfies only the natural conditions, and in particular, can have practically an arbitrary behaviour at the neighbourhood of 0 and $+\infty$ ([2]).

\section{The main result}

We deal with Orlicz classes $\varphi(L)$ of measurable $2 \pi$-periodic functions $f(x)$, such that the functional

$$
\|f\|_{\varphi}=\int_{0}^{2 \pi} \varphi(f(x)) d x
$$

is finite. Henceforth, $\varphi(x)$ is even, continuous, strictly monotonically increasing on $[0,+\infty)$ function, such that $\varphi(0)=0$ and

$$
\varphi(2 x) \leq C_{\varphi} \cdot \varphi(x), x \geq 0
$$

for some positive constant $C_{\varphi}$. The condition (1) is quite natural. In particular, it provides linearity of the class $\varphi(L)$.

For $f(x) \in \varphi(L)$ we define its modulus of continuity

$$
\omega(f, \delta)_{\varphi}=\sup _{0 \leq h \leq \delta}\|f(x+h)-f(x)\|_{\varphi}, \delta \geq 0,
$$

and its best approximation

$$
E_{n}(f)_{\varphi}=\inf _{T_{n}}\left\|f-T_{n}\right\|_{\varphi}, n=0,1,2, \ldots
$$


by trigonometric polynomials $T_{n}$ of order at most $n$. If $\varphi(x)=|x|^{p}$ for a certain $p>0$, the Jackson type inequality holds, that is,

$$
E_{n}(f)_{p} \leq C_{p} \cdot \omega\left(f, \frac{1}{n+1}\right)_{p}, n=0,1, \ldots, f \in L_{p},
$$

where the positive constant $C_{p}$ does not depend on $f$ and $n$.

Before we formulate the main result of this paper we introduce a concept of non-localized system. Let $e$ be a measurable subset of real axis with positive Lebesgue measure $\mu(e)$. As usual, we denote by symbol $L_{\infty}(e)$ a space of essentially bounded measurable functions equipped with the norm

$$
\|f\|_{L_{\infty}(e)}=\operatorname{ess} \sup |f(x)|, f \in L_{\infty}(e) .
$$

Definition. A system $\Omega=\left\{\omega_{n}\right\}_{n=1}^{+\infty}$ of functions in $L_{\infty}$ is non-localized, if there exist $\delta \in(0, \pi)$ and a sequence of positive numbers $\left\{a_{n}\right\}_{n=1}^{+\infty}$, such that

$$
\|f\|_{\infty} \leq a_{n} \cdot \inf \left\{\|f\|_{L_{\infty}(e)}: e \subset[0,2 \pi), \mu(e)>2(\pi-\delta)\right\},
$$

for all $f \in \Omega_{n}=\operatorname{span}\left\{\omega_{1}, \ldots, \omega_{n}\right\}$ and $n \in \mathbb{N}$.

Clearly, Haar system does not satisfy this definition. Afterwards we will prove that the trigonometric system does.

Theorem. Let $\varphi(x)$ satisfy the conditions above and $\Omega$ be a non-localized system. If

$$
\text { (A) } x^{p}=O(\varphi(x)), x \rightarrow+0 \text { for every } p>0
$$

or

$$
\text { (B) } \varphi(x)=O\left(x^{p}\right), x \rightarrow+\infty \text { for every } p>0 \text {, }
$$

then for each sequence of positive numbers $\left\{\sigma_{n}\right\}_{n=1}^{+\infty}$ that converges to 0 and for each positive constant $C$ there exist $f(x)$ in $\varphi(L)$ and $n \in \mathbb{N}$, such that

$$
E_{n}(f)_{\varphi ; \Omega}>C \omega\left(f, \sigma_{n}\right)_{\varphi} .
$$


Here

$$
E_{n}(f)_{\varphi ; \Omega}=\inf _{g \in \Omega_{n}}\|f-g\|_{\varphi}, n \in \mathbb{N}
$$

is a best approximation to $f$ in $\varphi(L)$ by polynomials with respect to the system $\Omega$ and as usual, " $g(x)=O(h(x)), x \rightarrow+0(x \rightarrow+\infty)$ " means that there exist a positive constant $C$ and $X>0$, such that $|g(x)| \leq C|h(x)|$ for $x \in(0, X)((X,+\infty))$.

\section{Proofs}

A proof of the Theorem is based on two Lemmas. We consider the function of "getting-out a constant" given by

$$
\Psi_{\varphi}(\lambda)=\sup _{x>0} \frac{\varphi(\lambda x)}{\varphi(x)}, \lambda \in \mathbb{R} .
$$

Because of (1) $\Psi_{\varphi}(\lambda)$ is well-defined. Clearly, it is monotonically increasing on $[0, \infty)$, even and $\Psi_{\varphi}(0)=0$.

Lemma 1. If $\varphi(x)$ satisfies $(A)$ or $(B), \Psi_{\varphi}(\lambda)=1$ for $\lambda \in(0,1]$.

Proof. First we consider the operator $S$ defined on a set of positive on $[0,+\infty)$ functions by

$$
S: g(x) \rightarrow\left(g\left(x^{-1}\right)\right)^{-1}
$$

and we notice that

$$
\Psi_{S \varphi}(\lambda)=\Psi_{\varphi}(\lambda), \lambda \in \mathbb{R}
$$

Moreover, $S=S^{-1}$ and condition (A) for $\varphi(x)$ is equivalent to condition (B) for $S \varphi(x)$. Therefore, it is sufficient to prove Lemma 1 only for one of them.

Let, for example, $\varphi(x)$ satisfy (B). Then for each $p>0$ there exist $C_{p}>0$ and $X_{p}>0$, such that

$$
\varphi(x) \leq C_{p} \cdot x^{p / 2}, x \in\left(X_{p},+\infty\right)
$$


and

$$
\frac{\ln \varphi(x)}{\ln x} \leq \frac{p}{2}+\frac{\ln C_{p}}{\ln x}, x \in\left(X_{p},+\infty\right)
$$

Therefore,

$$
\lim _{x \rightarrow+\infty} \varepsilon(x)=0, \quad\left(\varepsilon(x)=\frac{\ln \varphi(x)}{\ln x}\right) .
$$

As $\varepsilon(x)$ is positive and continuous on $[2,+\infty)$, we have from (5) that $\varepsilon_{0}=\sup _{x \in[2,+\infty)} \varepsilon(x) \in(0,+\infty)$. We set

$$
\mathcal{A}_{n}=\left\{x \in[2,+\infty): \varepsilon(x) \geq n^{-1}\right\}, n \geq n_{1},
$$

where $n_{1}=\left[\varepsilon_{0}^{-1}\right]+1$. Clearly, $\mathcal{A}_{n}$ are not empty. For each $\lambda \in(0,1)$ we consider the sequence

$$
t_{n} \equiv t_{n ; \lambda}=\lambda^{-1} \cdot \sup \mathcal{A}_{n}, n \geq n_{1} .
$$

Using (5), we have $t_{n}<+\infty, n \geq n_{1}$. Moreover,

$$
\text { 1) } \left.\lim _{n \rightarrow+\infty} t_{n}=+\infty ; 2\right) \varepsilon\left(\lambda t_{n}\right)>\varepsilon\left(t_{n}\right), n \geq n_{1} .
$$

Indeed, if $\lim _{n \rightarrow+\infty} t_{n}=t_{0}<+\infty$, any $x \in\left(t_{0},+\infty\right)$ does not belong to $\mathcal{A}_{n}$ for all $n \geq n_{1}$ and, therefore, $\varepsilon(x)=0$ and $\varphi(x)=1$ in $\left[t_{0},+\infty\right)$. To prove 2), we notice that $\varepsilon\left(\lambda t_{n}\right)=n^{-1}$ and $t_{n}>\lambda t_{n}=\sup \mathcal{A}_{n}$; therefore, $t_{n} \notin \mathcal{A}_{n}$ and $\varepsilon\left(t_{n}\right)<n^{-1}=\varepsilon\left(\lambda t_{n}\right)$.

Using 1), 2) and (5), we obtain

$$
\begin{aligned}
\Psi_{\varphi}(\lambda) & =\sup _{x>0} \frac{\varphi(\lambda x)}{\varphi(x)} \geq \sup _{n} \frac{\varphi\left(\lambda t_{n}\right)}{\varphi\left(t_{n}\right)}=\sup _{n} \exp \left\{\ln \varphi\left(\lambda t_{n}\right)-\ln \varphi\left(t_{n}\right)\right\} \\
& =\sup _{n}\left(\exp \left\{\ln t_{n} \cdot\left(\varepsilon\left(\lambda t_{n}\right)-\varepsilon\left(t_{n}\right)\right)\right\} \cdot \exp \left\{\ln \lambda \cdot \varepsilon\left(\lambda t_{n}\right)\right\}\right) \\
& \geq \sup _{n} \exp \left\{\ln \lambda \cdot \varepsilon\left(\lambda t_{n}\right)\right\} \geq \lim _{n \rightarrow+\infty} \exp \left\{\ln \lambda \cdot \varepsilon\left(\lambda t_{n}\right)\right\}=1 .
\end{aligned}
$$

The upper estimate is obvious.

The proof of Lemma 1 is complete. 
Lemma 2. Let $\Omega$ be a non-localized system. If there exist a sequence of positive numbers $\left\{\sigma_{n}\right\}_{n=1}^{+\infty}$ that converges to 0 and a positive constant $C$, such that

$$
E_{n}(f)_{\varphi ; \Omega} \leq C \omega\left(f, \sigma_{n}\right)_{\varphi}
$$

for all $f \in \varphi(L)$ and $n \in N$, then

$$
\lim _{\lambda \rightarrow+0} \Psi_{\varphi}(\lambda)=0
$$

Proof. Let $\Omega=\left\{\omega_{n}\right\}_{n=1}^{+\infty}$ be a non-localized system and $\delta>0$ as in (3). For each $\tau>0$ we consider a $2 \pi$-periodic function $f_{\tau}(x)$ that is equal to 0 for $x \in[0,2 \pi-\delta]$ and is equal to $\tau$ for $(2 \pi-\delta, 2 \pi)$. Let $n_{1}$ be a natural number, such that $\sigma_{n}<\delta$ for $n \leq n_{1}$. Clearly,

$$
\omega\left(f_{\tau}, \sigma_{n}\right)_{\varphi}=2 \varphi(\tau) \sigma_{n}, n \geq n_{1} .
$$

Actually,

$$
\begin{aligned}
\omega\left(f_{\tau}, \sigma_{n}\right)_{\varphi} & =\sup _{0 \leq h \leq \sigma_{n}} \int_{0}^{2 \pi} \varphi\left(f_{\tau}(x+h)-f_{\tau}(x)\right) d x \\
& =\sup _{0 \leq h \leq \sigma_{n}}\left\{\int_{0}^{2 \pi-\delta-h}+\int_{2 \pi-\delta-h}^{2 \pi-\delta}+\int_{2 \pi-\delta}^{2 \pi-h}+\int_{2 \pi-h}^{2 \pi}\right\} \\
& =\sup _{0 \leq h \leq \sigma_{n}} 2 h \varphi(\tau)=2 \varphi(\tau) \sigma_{n} .
\end{aligned}
$$

We choose $g_{n ; \tau}(x) \in \Omega_{n}$, such that

$$
\left\|f_{\tau}-g_{n ; \tau}\right\|_{\varphi} \leq E_{n}\left(f_{\tau}\right)_{\varphi ; \Omega}+C \omega\left(f, \sigma_{n}\right)_{\varphi}, n \geq n_{1} .
$$

Then we have from (6) and (7)

$$
\left\|f_{\tau}-g_{n ; \tau}\right\|_{\varphi} \leq 4 C \sigma_{n} \varphi(\tau), n \geq n_{1} .
$$

We set

$$
\mathcal{E} \equiv \mathcal{E}_{n ; \tau}=\left\{x \in[0,2 \pi-\delta): \varphi\left(g_{n ; \tau}(x)\right) \leq 4 C \delta^{-1} \sigma_{n} \varphi(\tau)\right\}, n \geq n_{1}, \tau>0
$$


To estimate their measures we use Chebyshev inequality and (8)

$$
\begin{aligned}
\mu(\mathcal{E}) & =2 \pi-\delta-\mu\left\{x \in[0,2 \pi-\delta): \varphi\left(g_{n ; \tau}(x)\right)>4 C \delta^{-1} \sigma_{n} \varphi(\tau)\right\} \\
& \geq 2 \pi-\delta-\left(4 C \delta^{-1} \sigma_{n} \varphi(\tau)\right)^{-1} \cdot \int_{0}^{2 \pi-\delta} \varphi\left(g_{n ; \tau}(x)\right) d x \\
& \geq 2 \pi-\delta-\left(4 C \delta^{-1} \sigma_{n} \varphi(\tau)\right)^{-1} \cdot\left\|f_{\tau}-g_{n ; \tau}\right\|_{\varphi} \geq 2(\pi-\delta) .
\end{aligned}
$$

Therefore

$$
\left\|g_{n ; \tau}\right\|_{\infty} \leq a_{n} \cdot\left\|g_{n ; \tau}\right\|_{L_{\infty}(\mathcal{E})} \leq \gamma_{n ; \tau}, n \geq n_{1}, \tau>0
$$

where

$$
\gamma_{n ; \tau}=a_{n} \cdot \varphi^{-1}\left(4 C \delta^{-1} \sigma_{n} \varphi(\tau)\right)
$$

Without loss of generality we can assume that $\left\{a_{n}\right\}_{n=1}^{+\infty}$ tends to $+\infty$.

Let $n_{2}$ be a natural number, such that

$$
\sigma_{n}^{-1}>4 C \delta^{-1} \Psi(2) \geq \frac{4 C \delta^{-1} \varphi(\tau)}{\varphi\left(\frac{\tau}{2}\right)}
$$

for all $n \geq n_{2}$ and $\tau>0$. Let also $n_{3}=\max \left\{n_{1}, n_{2}\right\}$. We will prove that

$$
\tau \leq 2 \gamma_{n ; \tau}
$$

for $n \geq n_{3}$ and $\tau>0$. Indeed, otherwise, by virtue of (9) we get for some $n \geq n_{3}$

$$
\tau-g_{n ; \tau}(x) \geq \tau-\left|g_{n ; \tau}(x)\right| \geq \tau-\gamma_{n ; \tau}>\frac{\tau}{2}
$$

almost everywhere in $[0,2 \pi)$. Furthermore,

$$
\left\|f_{\tau}-g_{n ; \tau}\right\|_{\varphi} \geq \int_{2 \pi^{-} \delta}^{2 \pi} \varphi\left(\tau-g_{n ; \tau}(x)\right) d x \geq \delta \cdot \varphi\left(\frac{\tau}{2}\right)>4 C \sigma_{n} \varphi(\tau)
$$

that is in contradiction with (8). 
We rewrite (10) as follows:

$$
\begin{gathered}
\tau \leq 2 a_{n} \varphi^{-1}\left(4 C \delta^{-1} \sigma_{n} \varphi(\tau)\right) \\
\frac{\varphi\left(\left(2 a_{n}\right)^{-1} \tau\right)}{\varphi(\tau)} \leq 4 C \delta^{-1} \sigma_{n}, \tau>0, n \geq n_{3} .
\end{gathered}
$$

As the right-hand side of (11) does not depend on $\tau$, we get

$$
\Psi_{\varphi}\left(\left(2 a_{n}\right)^{-1}\right) \leq 4 C \delta^{-1} \sigma_{n}, n \geq n_{3} .
$$

Thus, there exists a sequence $\lambda_{n}=\left(2 a_{n}\right)^{-1}, n \geq n_{3}$, that converges to 0 and $\lim _{n \rightarrow+\infty} \Psi_{\varphi}\left(\lambda_{n}\right)=0$. As $\Psi_{\varphi}(\lambda)$ increases on $[0,+\infty)$, we have finally

$$
\lim _{\lambda \rightarrow+0} \Psi_{\varphi}(\lambda)=0
$$

Lemma 2 is proved.

Proof of Theorem. Theorem follows immediately from Lemmas 1 and 2 .

\section{Non-locality of systems of analytic functions}

Now we prove that a system of $2 \pi$-periodic analytic functions is nonlocalized. Without loss of generality we can assume that it is linearly independent. We consider the functions

$$
\begin{aligned}
& \Phi(\bar{\lambda})=\inf \left\{\left\|\lambda_{1} \omega_{1}+\ldots+\lambda_{n} \omega_{n}\right\|_{L_{\infty}(e)}: e \subset[0,2 \pi), \mu(e) \geq \pi\right\} ; \\
& F(\bar{\lambda})=\left\|\lambda_{1} \omega_{1}+\ldots+\lambda_{n} \omega_{n}\right\|_{\infty}, \bar{\lambda}=\left(\lambda_{1} \ldots \lambda_{n}\right) \in \mathbb{R}^{n} .
\end{aligned}
$$

It is easy to see that they are continuous on the sphere $\mathcal{S}^{n-1}=$ $\left\{\bar{\lambda} \in \mathbb{R}^{n}: \lambda_{1}^{2}+\ldots+\lambda_{n}^{2}=1\right\}$.

Furthermore,

$$
\Phi(\bar{\lambda}) \neq 0, \bar{\lambda} \in \mathcal{S}^{n-1}
$$


Indeed, if $\Phi(\bar{\lambda})=0$ for a certain $\bar{\lambda} \in \mathcal{S}^{n-1}$, there exists a sequence of measurable sets $\left\{e_{m}\right\}_{m=1}^{+\infty}$, such that $e_{m} \subset[0,2 \pi), \mu\left(e_{m}\right) \geq \pi$ and

$$
\|g\|_{L_{\infty}\left(e_{m}\right)} \leq m^{-1}, m \in \mathbb{N},
$$

where $g \equiv \lambda_{1} \omega_{1}+\ldots+\lambda_{n} \omega_{n}$. Let

$$
\mathcal{E}_{m}=\left\{x \in[0,2 \pi):|g(x)| \leq m^{-1}\right\}, m \in \mathbb{N} .
$$

Clearly,

$$
\mathcal{E}_{m} \supseteq e_{m}, m \in \mathbb{N} ; \mathcal{E}_{1} \supseteq \mathcal{E}_{2} \supseteq \ldots \supseteq \mathcal{E}_{m} \supseteq \ldots
$$

Hence,

$$
\mu(\mathcal{E})=\lim _{m \rightarrow+\infty} \mu\left(\mathcal{E}_{m}\right) \geq \lim _{m \rightarrow+\infty} \mu\left(e_{m}\right) \geq \pi, \quad\left(\mathcal{E}=\bigcap_{m=1}^{+\infty} \mathcal{E}_{m}\right) .
$$

As $g(x)=0, x \in \mathcal{E}$, we have by the uniqueness theorem for analytic functions that $g(x)=0$ and, therefore, $\bar{\lambda}=0$, that is in contradiction with the condition: $\bar{\lambda} \in S^{n-1}$.

By virtue of (12) the function $\frac{F(\bar{\lambda})}{\Phi(\lambda)}$ is continuous on $\mathcal{S}^{n-1}$. As it is homogenous, there exists a sequence of positive numbers $\left\{a_{n}\right\}_{n=1}^{+\infty}$, such that

$$
F(\bar{\lambda}) \leq a_{n} \Phi(\bar{\lambda}), \quad \bar{\lambda} \in \mathbb{R}^{n}
$$

\section{Remarks}

1. As it follows from our research, the class of functions $\varphi(x)$, for which Jackson type inequality is valid, is being practically exhausted by power functions. It means that there does not exist a "bound" function as it has been believed. However, it should be noted, that the powers near 0 and $+\infty$ can be different from each other.

2. As is known, if $\varphi_{1}(x)=\varphi_{2}(x)$ for some $x_{0}>0$, then $\varphi_{1}(L)=$ $\varphi_{2}(L)$ and convergences in these classes are equivalent to each other. It is obvious that if we change $\varphi(x)$ in any segment $\left[x_{1}, x_{2}\right]$, where $0<x_{1}<x_{2}<+\infty$, this does not affect the validity of Jackson type inequality. However, in difference from the topological properties of Orlicz 
classes it depends essentially on behaviour of $\varphi(x)$ near 0 . Besides, the contributions of 0 and $+\infty$ turn out to be similar.

3. It can be shown by the method developed in [3] that a converse result to Lemma 2 is valid, if $\varphi(x+y) \leq \varphi(x)+\varphi(y)$ for $x, y \geq 0$. However, we do not regard it to be very important, because as it follows from Lemma 1, the function of "getting-out a constant" is equal to 1 , if $\varphi(x)$ is slower than any power function near 0 or $+\infty$. Moreover, if $\varphi(x)$ is a power function, $\Psi_{\varphi}(x)=\varphi(x)$. Thus, the situation: $\lim _{\lambda \rightarrow+0} \Psi_{\varphi}(\lambda)=$ 0 turns out to be interesting only for functions, which have "abnormal" behaviour.

\section{References}

[1] H. Shapiro, "Topics in Approximation Theory", Lecture Notes in Mathematics, Vol. 187, Springer-Verlag, 1970.

[2] E.A. Storozhenko, V. Krotov, P. Oswald, Direct and converse theorems of Jackson type in $L_{p}$-spaces, $0<p<1$, Math. USSR-Sb., 27 (3) (1975).

[3] K. Runovski, On approximation by families of linear polynomial operators in $L_{p}$-spaces, $0<p<1$, Russian Acad. Sci. Sb. Math., Vol. 82 (1995), No. 2 pp. $441-459$.

Mathematisches Institut

Friedrich-Schiller-Universität-Jena

Ernst-Abbe-Platz, 1-4, 07743 Jena Germany

E-mail: runovski@informatik.uni-jena.de

Recibido: 23 de Noviembre de 2000

Revisado: 4 de Diciembre de 2000 